\title{
Evidências de Validade da Escala de Autoeficácia da Bateria de Avaliação
}

\author{
de Indicadores da Depressão Infantojuvenil
}

\author{
Daiane Nunes* \\ Universidade Federal de Sergipe - UFS, São Cristovão, SE, Brasil \\ ORCID: https://orcid.org/0000-0002-8680-0206 \\ Laís Santos** \\ Pontifícia Universidade Católica de Campinas - PUC-Campinas, Campinas, SP, Brasil \\ ORCID: https://orcid.org/0000-0001-7246-7476 \\ André Faro*** \\ Universidade Federal de Sergipe - UFS, São Cristovão, SE, Brasil \\ ORCID: https://orcid.org/0000-0002-7348-6297 \\ Makilim Nunes Baptista***** \\ Universidade Federal de Sergipe - UFS, São Cristovão, SE, Brasil \\ ORCID: https://orcid.org/0000-0001-6519-254X
}

\section{RESUMO}

Este estudo analisou evidências de validade da Escala de Autoeficácia da Bateria de Avaliação de Indicadores da Depressão Infatojuvenil, avaliou a validade divergente da escala em relação à depressão e examinou a distribuição social da autoeficácia segundo as variáveis sexo, idade e ano escolar. A amostra consistiu de 388 adolescentes, com idades entre 14 e 19 anos que responderam a um questionário sociodemográfico e à Escala de Autoeficácia. A Análise Fatorial Exploratória foi conduzida por meio do software Factor. Os resultados indicaram a unidimensionalidade da medida, explicando 56,3\% de variância. Os índices de ajuste do modelo e o índice de confiabilidade foram satisfatórios. A avaliação da distribuição social mostrou que houve relações estatisticamente significativas quanto ao sexo e a idade. Constatou-se que a Escala de Autoeficácia apresentou boas propriedades psicométricas, o que recomenda seu uso por parte da comunidade científica e profissional para futuras pesquisas, intervenções e avaliações da autoeficácia em adolescentes.

Palavras-chave: autoeficácia, escala de autoeficácia, validade do teste, psicometria.

\section{Validity Evidences of Evaluation Battery for Childhood Depression}

\author{
Indicators Self-efficacy Scale
}

\begin{abstract}
This study analyzed validity evidences of the Evaluation Battery for Childhood Depression Indicators Self-efficacy Scale, assessed the divergent validity of the scale in relation to
\end{abstract}


depression and examined the social distribution of self-efficacy according to gender, age, and school grade. The sample consisted of 388 adolescents, aged between 14 and 19 years old. It was administered a sociodemographic questionnaire and the Self-efficacy Scale. Exploratory Factor Analysis was conducted using the Factor software. The results indicated the unidimensionality of the scale, explained $56.3 \%$ of the total variance. The fit indices of the model and the reliability index were satisfactory. The evaluation of the social distribution showed statistically significant relationships between self-efficacy, sex and age. Finally, the Self-efficacy Scale showed satisfactory psychometric properties, which recommend its use by the scientific and professional community for future research, interventions, and evaluations of the self-efficacy in adolescents.

Keywords: self-efficacy, self-efficacy scale, test validity, psychometrics.

\title{
Evidencias de Validez de la Escala de Autoeficacia de la Batería de
}

\section{Evaluación de Indicadores de la Depresión Infantojuvenil}

\begin{abstract}
RESUMEN
Este estudio analizó evidencias de validez de la Escala de Autoeficacia de la Batería de Evaluación de Indicadores de la Depresión Infantojuvenil, evaluó la validez divergente de la escala en relación a la depresión y examinó la distribución social de la autoeficacia según las variables sexo, edad y grado escolar. La muestra consistió de 388 adolescentes entre 14 y 19 años. Fueron empleados un cuestionario sociodemográfico y la Escala de Autoeficacia. El Análisis Factorial Exploratorio fue conducido por medio del software Factor. Los resultados revelaron la unidimensionalidad de la medida, explicando 56,3\% de la varianza. Los índices de ajuste del modelo y de confiabilidad fueron satisfactorios. La evaluación de la distribución social mostró que hubo relaciones estadísticamente significativas entre autoeficacia, sexo y edad. Finalmente, se constató que la Escala de Autoeficacia presentó buenas propiedades psicométricas, lo que recomienda su uso por parte de la comunidad científica y profesional para futuras investigaciones y evaluaciones de la autoeficacia de adolescentes.
\end{abstract}

Palabras clave: autoeficacia, escala de autoeficacia, validez de la prueba, psicometría.

A adolescência é um período marcado por intensas mudanças de natureza biopsicossocial, incluindo modificações corporais e adaptação a novas estruturas psicológicas. Essas mudanças tornam a adolescência um período importante no curso de vida e para o estabelecimento das bases de saúde na vida adulta (Jager, Batista, Perrone, Santos, \& Dias, 2014). Muitos comportamentos classificados como de risco à saúde, que geralmente têm início nessa fase (por exemplo, tabagismo, consumo de álcool e drogas ilícitas), são responsáveis pelo aumento da morbidade, mortalidade e contribuem para o desenvolvimento 
de doenças como a hipertensão e diabetes (Sawyer et al., 2012; World Health Organization [WHO], 2014). Além disso, mais de 50\% da ocorrência de transtornos mentais na vida adulta têm início antes dos 18 anos de idade, produzindo desordens crônicas e uma série de dificuldades psicossociais (Das et al., 2016; Jones, 2013). Portanto, evidencia-se a necessidade de incluir aspectos psicológicos no entendimento em saúde nesse grupo, já que estes estão diretamente relacionados ao desencadeamento de problemas na saúde física (Capitão, Scortegagna, \& Baptista, 2005).

Variáveis psicológicas como a resiliência (Sánchez-Teruel \& Robles-Bello, 2015), autoestima (Gao, Zhang, \& Xu, 2015), autoconceito (Galvéz-Nieto, Polanco, \& Salvo, 2017) e autoeficácia (Ma, Zeng, \& Ye, 2015; Orcasita, Gil, \& González, 2018; Valois, Zullig, \& Hunter, 2015), apresentam-se como elementos protetivos e auxiliam no processo de ajustamento psicológico dos indivíduos. Nesse contexto, a autoeficácia tem chamado a atenção diante das evidências de que esta influencia significativamente a adoção, iniciação e manutenção de comportamentos de saúde (Schnell, Ringeisen, Raufelder, \& Rohrmann, 2015; Sheeran et al., 2016). O construto tem sido apontado como um importante preditor para diferentes comportamentos em saúde e condições clínicas, como na adesão à terapia antirretroviral para o HIV/AIDS (Mao et al., 2018), ao tratamento para Diabetes Tipo 2 (Santos \& Faro, 2018), ao tratamento medicamentoso em pacientes hipertensos (Ahn \& Ham, 2016) e à prática de atividade física (Selzler, Rodgers, Berry, \& Stickland, 2020). Na saúde mental, a autoeficácia se mostrou como um relevante mediador para a depressão (SerraTaylor, \& Irizarry-Robles, 2015), ansiedade (Recber, Isiksal, \& Koç, 2018) e transtorno de estresse pós-traumático (Guerra, Farkas, \& Moncada, 2018).

Originalmente proposto por Bandura (1977), a autoeficácia refere-se às crenças do indivíduo acerca de sua capacidade em organizar e executar cursos de ação requeridos para produzir certos resultados. Na Psicologia da Saúde, as crenças de autoeficácia são cognições que influenciam comportamentos em saúde, determinam quanto esforço será empreendido e quanto tempo tais comportamentos serão mantidos diante de obstáculos e fracassos (Bandura, 1986). Indivíduos com alta percepção de autoeficácia estabelecem metas mais complexas e aderem melhor a elas, investindo mais esforços e persistindo por mais tempo do que aqueles com baixa percepção de autoeficácia (Schwarzer \& Luszczynska, 2006). As crenças de autoeficácia são desenvolvidas a partir de uma série de mecanismos cognitivos, sociais e, inclusive, fisiológicos. Os indivíduos associam excitação fisiológica aversiva com desempenho comportamental ruim, reforçando crenças de incompetência. Por sua vez, estados fisiológicos positivos tendem a potencializar níveis elevados de autoeficácia (Bandura, 1986). 
$\mathrm{Na}$ adolescência, as crenças de autoeficácia podem atuar no gerenciamento de emoções e relações interpessoais, colaborando na promoção de expectativas positivas sobre o futuro. Além disso, auxilia na manutenção de um autoconceito mais positivo e na satisfação pela vida, produzindo emoções mais positivas frente às demandas adaptativas inerentes à fase (Mesurado, Vidal, \& Mestre, 2018). Intervenções educacionais com foco nos efeitos da variável no bem-estar psicológico se demonstraram proficientes na capacitação de recursos pessoais (Tak, Brunwasser, Lichtwarck-Aschoff, \& Engels, 2017) e regulação emocional (Soysa \& Wilcomb, 2015). Altos níveis de sintomas depressivos (Guerra et al., 2018; Muris, Meesters, Pierik, \& Kock, 2016; Yang, Lau, \& Lau, 2018) e de ansiedade (Raknes et al., 2017) também foram associados à baixa autoeficácia em adolescentes. Tais achados evidenciam a importância de intervenções com o foco no fortalecimento de crenças de autoeficácia mais adaptativas e a necessidade de aperfeiçoamento dos processos investigativos da variável nessa população (Mesurado et al., 2018).

Os níveis de autoeficácia podem variar de acordo com uma série de características individuais dos adolescentes e do ambiente, incluindo sua faixa etária (Ndika, Olagbaiye, \& Agiobu-Kemmer, 2009), sexo (Johnson \& Muse, 2017) e desafios impostos pelo contexto escolar, como nível de escolaridade (Joseph, Anikelechi, \& Marumo, 2019). Não há um consenso na literatura acerca das diferenças nos níveis de autoeficácia quanto à idade do adolescente. Ora os mais novos apresentam níveis mais rebaixados de autoeficácia, ora os mais velhos. Por vezes, ainda, não se constata uma relação estatisticamente significativa entre autoeficácia e faixa etária do adolescente (Ndika et al., 2009). O mesmo pode ser afirmado quanto ao ano escolar em que este se encontra (Joseph et al., 2019). Acerca da variável sexo, estudos indicam que as diferenças nos níveis de autoeficácia entre meninos e meninas são mais bem explicadas quando associadas ao contexto ou domínio. De modo geral, as meninas apresentam níveis mais elevados de autoeficácia para atividades como escrita e leitura, comparativamente aos meninos, que tendem a demonstrar crenças mais elevadas em matemática e tecnologias (Johnson \& Muse, 2017). Assim, mais investigações se tornam necessárias para o esclarecimento das diferenças individuais nos níveis de autoeficácia.

A avaliação de autoeficácia tem sido realizada através da Escala de Autoeficácia Geral Percebida - EAGP (General Perceived Self-efficacy Scale; Schwarzer \& Jerusalem, 1995) e da Escala de Autoeficácia Geral - EAG (General Self-Efficacy Scale; Sherer et al., 1982), sendo apenas a primeira validada para o contexto brasileiro (Sbicigo, Teixeira, Dias, \& Dell'Aglio, 2012). Trata-se de medidas gerais de autoeficácia, que não foram construídas especificamente para a investigação da variável em crianças e adolescentes. 
O Self-Efficacy Questionnaire for Children (SEQ-C) (Muris, 2001) foi construído e validado para mensurar a percepção de autoeficácia de crianças e adolescentes em três domínios: social, acadêmico e emocional, estabelecidos a partir de Bandura, Pastorelli, Barbaranelli e Caprara (1999). A autoeficácia social refere-se à capacidade dessa população em lidar com os desafios sociais; a acadêmica remete à capacidade para dominar assuntos acadêmicos e escolares; e autorreguladora (emocional) está relacionada com o manejo de situações estressoras, como, por exemplo, a capacidade de resistir à pressão dos pares no envolvimento em atividades de risco (Bandura et al., 1999). Contudo, cabe ressaltar que não há evidências de validação e adaptação transcultural desse instrumento no contexto brasileiro.

Por sua vez, a Bateria de Avaliação de Indicadores da Depressão Infantojuvenil BAID-IJ (C) (Borges, Baptista, \& Serpa, 2017), construída e validada no contexto brasileiro, investiga depressão e indicadores a ela correlacionados, incluindo as subescalas de solidão, desamparo, autoestima, autoconceito, desesperança e autoeficácia, sendo esta última o objeto desse estudo. As subescalas da BAID-IJ são independentes entre si, possibilitando que cada indicador seja avaliado de forma individual e com a obtenção de um escore específico para cada uma delas (Borges, 2015).

A Escala de Autoeficácia foi construída a partir da teoria de Bandura (1986) sobre autoeficácia, entendendo que as crenças das pessoas sobre suas capacidades influenciam suas escolhas e afetam o quanto elas mobilizam, persistem e se engajam em padrões de pensamentos autodebilitantes ou autoencorajadores. Suas dimensões compreendem aspectos inerentes ao público infantojuvenil, a exemplo das crenças de eficácia acerca da relação social com pares e familiares (item 11- se relacionar bem com a sua minha família), do desempenho acadêmico (item 13- se dar bem nas avaliações escolares), e de capacidade autorreguladora (item 15- de conseguir sair bem de situações difíceis) (Cardoso, 2018).

A Escala de Autoeficácia é uma medida curta e simples e, portanto, de fácil aplicação. Trata-se de um instrumento construído e validado especificamente para o público infantojuvenil, inserido dentro de uma bateria de avaliação. Tal característica permite o rastreamento e a identificação de níveis de severidade dos sintomas de depressão, como o direcionamento do tratamento com base nos escores individuais de cada indicador, mostrando-se uma ferramenta eficaz na clínica (Cardoso, 2018).

O estudo de evidências de validade da estrutura interna da subescala de autoeficácia indicou a unidimensionalidade da medida, com propriedades psicométricas adequadas $(\alpha=$ 0,91). A Escala de Autoeficácia foi aplicada em uma amostra de crianças e adolescentes, com idades entre oito e 18 anos, proveniente do Estado do Rio Grande do Sul (Cardoso, 2018), 
carecendo, então, de mais estudos que evidenciem suas qualidades psicométricas em outras regiões do Brasil e com amostras de adolescentes.

Diante da necessidade de apresentar um aparato instrumental com boas propriedades psicométricas para auxiliar nas práticas acadêmicas e profissionais, este estudo objetivou apresentar evidências de validade da Escala de Autoeficácia da BAID-IJ, por meio de Análise Fatorial Exploratória (AFE) e de análise de validade divergente entre as variáveis autoeficácia e depressão. Além disso, analisou-se a distribuição social da autoeficácia segundo as variáveis sexo, idade e ano escolar.

\section{Método}

\section{Amostra}

O delineamento da pesquisa foi do tipo não probabilístico, com amostragem por conveniência. Participaram 388 jovens de 14 a 19 anos do ensino médio de escolas públicas de Aracaju $(n=248 ; 63,9 \%)$ e Itabaiana $(n=148 ; 38,1)$, que compuseram a amostra do presente estudo. A média de idade foi de 16,3 anos $(D P=1,17)$, com maioria do sexo feminino $(n=231 ; 59,5 \%)$. Com relação ao ano escolar, 49,7\% $(n=193)$ foram de estudantes do $1^{\circ}$ ano, $29,9 \%(n=116)$ do $2^{\circ}$ ano e $20,4 \%(n=79)$ do $3^{\circ}$ ano.

\section{Instrumentos}

Os instrumentos utilizados incluíram um questionário sociodemográfico, contendo informações como idade, sexo e escolaridade, a Escala de Autoeficácia e a Escala de Depressão da Bateria de Avaliação de Indicadores de Depressão Infantojuvenil (BAID-IJ) (Borges, Baptista, \& Serpa, 2017). A Escala de Autoeficácia é constituída de 15 itens respondidos numa escala tipo Likert de 3 pontos $(0=$ Não/Nunca, $1=$ Às vezes e $2=$ Sim/Sempre). O escore final é produzido pela soma dos itens, variando entre 0 e 30 pontos, em que quanto mais alto o escore, maior a percepção de autoeficácia da amostra. A escala de depressão, por sua vez, é formada por 18 itens e o escore final varia entre 0 e 36 pontos. Quanto mais alto o escore, maior a presença de sintomas de depressão. 


\section{Procedimentos}

Obteve-se a autorização das instituições, bem como foram informados aos responsáveis e aos adolescentes os objetivos e aspectos éticos da pesquisa, sendo então convidados a participarem após assinatura do Termo de Autorização dos Responsáveis e do Termo de Assentimento Livre e Esclarecido (TALE). Os instrumentos foram aplicados de forma presencial, com duração aproximada de 20 minutos. O estudo foi previamente submetido e aprovado pelo Comitê de Ética em Pesquisa com Seres Humanos (CAEE: 54236016.0.0000.5546; Número do parecer: 1.505.545).

\section{Análise dos Dados}

Inicialmente foi executada a análise exploratória das variáveis no Statistical Package for the Social Sciences (SPSS - versão 20), realizando-se a substituição de casos perdidos e extremos (menos do que $1 \%$ da amostra total) pela média, bem como a análise dos coeficientes de assimetria e achatamento. Em seguida, obtiveram-se as estatísticas descritivas (frequência relativa, média e desvios-padrão).

A estrutura interna da Escala de Autoeficácia da BAID-IJ foi analisada por meio de Análise Fatorial Exploratória (AFE) com o software Factor, versão 10.8 (Lorenzo-Seva \& Ferrando, 2006). O Factor permite a utilização de matrizes de correlação policórica, que são especialmente indicadas quando os itens se apresentam em formato de resposta tipo Likert. Optou-se por utilizá-lo, ainda, considerando a possibilidade de realização de análises mais robustas oferecidas, a exemplo de análises semiconfirmatórias (Ferrando \& Lorenzo-Seva, 2017). O método de estimação dos parâmetros foi o Diagonally Weighted Least Squares (DWLS), imputado quando os dados não requerem distribuição normal (Bollen, 1989) e rotação Promin. Para selecionar a quantidade de fatores a ser extraída, foi utilizada a Análise Paralela, bem como foi utilizado o parâmetro Closeness to Unidimensionality Assessment para verificar a plausibilidade de o instrumento ser tratado como unidimensional, considerando-se os índices esperados como UniCO > 0,95, ECV > 0,85 e MIREAL < 0,30.

Os índices de ajuste utilizados para a estimação de adequação do modelo foram o Comparative Fit Index (CFI) e o Non-Normed Fit Index (NNFI), com valores acima de 0,95 considerados aceitáveis, além do Root Mean Square Error of Approximation (RMSEA) < 0,080 (Hair, Black, Babin, Anderson, \& Tatham, 2009). Os índices de replicabilidade e fidedignidade utilizados foram, respectivamente, o H-latent e o alfa de Cronbach. 
Após a realização da AFE, foi conduzida no SPSS análise de correlação de Spearman $(\rho)$ para investigar a validade divergente entre o escore total da Escala de Autoeficácia e a escala de Depressão. Realizou-se, ainda, uma análise sobre a distribuição social da Autoeficácia segundo a variável sexo (teste $U$ de Mann-Whitney), a variável idade [correlação de Spearman $(\rho)$ ] e a variável ano escolar [teste de Kruskal-Wallis $\left(X^{2}\right)$ ].

\section{Resultados}

\section{Evidências de validade da Escala de Autoeficácia: Estrutura interna e validade divergente}

$\mathrm{Na}$ avaliação da estrutura interna da Escala de autoeficácia, o teste de esfericidade de Bartlett teve significância estatística $\chi_{(105)}^{2}=1686,7 ; p<0,001$ e o índice KMO foi de 0,882, sugerindo adequação para a realização da AFE. O teste de Mardia mostrou significância estatística para a curtose multivariada $(14,88, p<0,001)$, embora não para a assimetria multivariada, concluindo que a distribuição foi não normal. A AP sugeriu uma estrutura de um único fator, com variância explicada de 56,3\%. O Closeness to Unidimensionality Assessment, reforçou a unidimensionalidade da escala (UniCO = 0,94 e IC95\% = 0,92 - 0,97; $\mathrm{ECV}=0,83$ e IC95\% = 0,82 - 0,86; MIREAL $=0,22$ e IC95\% $=0,18-0,23$ ).

Demonstrada a fatorabilidade da escala, conduziu-se a AFE imputando-se apenas um fator indicado pela AP. Utilizou-se o critério de carga fatorial acima de 0,30. Neste modelo, as cargas fatoriais dos itens variaram entre 0,43 e $0,81(M=0,63 ; D P=0,10)$, de modo que nenhum item carregou abaixo do critério aplicado, mantendo-se assim a mesma estrutura da escala com 15 itens. O RMSEA foi de 0,07, ao passo que o NNFI e o CFI foram de 0,95, indicando boa qualidade do ajuste da escala aos dados. O H-latent foi abalizado como ótimo nesta solução $(0,95)$. O alfa de Cronbach teve o valor de 0,90 , considerado excelente. As informações da AFE constam na Tabela 1. 


\begin{tabular}{|c|c|c|c|}
\hline Itens da Escala de Autoeficácia & $M$ & $D P$ & $\lambda$ \\
\hline Item 1 & 1,7 & 0,49 & 0,43 \\
\hline Item 2 & 1,5 & 0,54 & 0,66 \\
\hline Item 3 & 1,4 & 0,55 & 0,69 \\
\hline Item 4 & 1,3 & 0,57 & 0,54 \\
\hline Item 5 & 1,3 & 0,56 & 0,70 \\
\hline Item 6 & 1,1 & 0,56 & 0,53 \\
\hline Item 7 & 1,3 & 0,62 & 0,62 \\
\hline Item 8 & 0,8 & 0,73 & 0,55 \\
\hline Item 9 & 1,2 & 0,63 & 0,74 \\
\hline Item 10 & 1,1 & 0,74 & 0,61 \\
\hline Item 11 & 1,5 & 0,61 & 0,60 \\
\hline Item 12 & 1,7 & 0,46 & 0,71 \\
\hline Item 13 & 1,2 & 0,49 & 0,56 \\
\hline Item 14 & 1,1 & 0,58 & 0,79 \\
\hline Item 15 & 1,2 & 0,59 & 0,81 \\
\hline
\end{tabular}

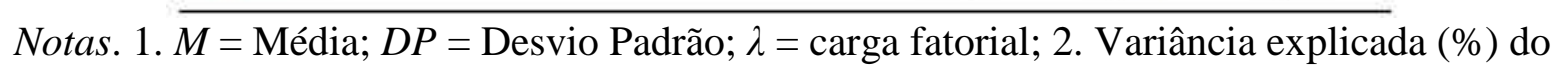
modelo final igual a 56,3\%; 3 . Indicadores de ajuste do modelo final. RMSEA = 0,07, NNFI $=0,95, \mathrm{CFI}=0,95 ; 4$, Indicadores de replicabilidade e confiabilidade: $H$-latent $=0,95$ e alfa de Cronbach $=0,90 ; 5$. Indicadores de unidimensionalidade: $\mathrm{UniCO}=0,94$ (IC95\% $=0,92-$ $0,97), \mathrm{ECV}=0,83(\mathrm{IC} 95 \%=0,82-0,86)$ e $\mathrm{MIREAL}=0,22(\mathrm{IC} 95 \%=0,18-0,23)$.

A fim de apresentar evidências de validade divergente, realizou-se teste de correlação de Spearman entre os escores totais nas Escalas de autoeficácia e de depressão. O resultado da matriz de correlação indicou uma associação estatisticamente significativa, moderada e negativa $(r=-0,506 ; p<0,001)$ entre ambos os escores.

\section{Distribuição Social da Escala de Autoeficácia da BAID-IJ}

Para avaliação da distribuição social da autoeficácia, obteve-se, inicialmente, a mediana do escore total na amostra $(M d=20,0 ;$ Mínimo $=6,0 ;$ Máximo $=30,0)$, demonstrando que esta tende a perceber sua autoeficácia acima do ponto médio da escala (pontos15). Vale ressaltar que quanto mais alto o escore, maior a percepção da amostra acerca 
do construto. Posteriormente, estratificou-se a variável autoeficácia em três grupos a partir da distribuição do escore total da presente amostra, nomeadas como: "baixa autoeficácia" (0 a 17 pontos), com 30,2\% ( $n=117)$, "moderada autoeficácia" (18 a 21 pontos), com 31,2\% ( $n=$ 121), e "elevada autoeficácia" (22 a 30 pontos), 38,7\% $(n=150)$.

Foi utilizado o teste $U$ de Mann-Whitney e a correlação de Spearman $(\rho)$ para investigar como as medianas obtidas no escore total de Autoeficácia se associavam às variáveis sociodemográficas sexo e idade. O resultado do teste $U$ revelou diferença estatisticamente significativa entre meninos $(M d=22,0)$ e meninas $(M d=19,0)(U=-4,07 ; p$ $<0,001)$. A correlação foi negativa e significativa com a variável idade $(r=-0,25 ; p<0,001)$. O resultado do teste Kruskal-Wallis não apresentou um efeito significativo em relação à variável ano escolar e à autoeficácia $\left(X^{2}=2,20 ; p=0,332\right)$.

\section{Discussão}

O presente estudo teve como objetivo realizar AFE da Escala de Autoeficácia da BAID-IJ, bem como analisar a distribuição social da autoeficácia segundo as variáveis sexo e idade e ano escolar. A análise indicou a unidimensionalidade da escala e apresentou propriedades psicométricas adequadas para a avaliação do construto que investiga. Os indicadores de confiabilidade, a partir do valor do alfa de Cronbach $(0,90)$ e, de replicabilidade a partir do $H$-latent $(0,95)$, atenderam o esperado, demonstrando estabilidade da estrutura latente da escala (Ferrando \& Lorenzo-Seva, 2017).

As cargas fatoriais obtidas foram adequadas, todas acima do ponto de corte recomendado pela literatura $(0,30)(M=0,63 ; D P=0,10)$, assim como a variância explicada que foi de 56,3\%, valor este considerado bom para pesquisas da área de humanas e, em especial, na Psicologia (Damásio, 2012). Por sua vez, o estudo original realizado por Cardoso (2018) em amostra do Sul do Brasil, obteve média das cargas fatoriais de 0,61 $(D P=0,10)$ e variância explicada equivalente a 41,0\%. Portanto, os resultados deste estudo, com amostra do Nordeste brasileiro, apontaram evidências de validade da estrutura interna e confiabilidade do instrumento, tendo sido superiores, inclusive, aos indicadores obtidos no Rio Grande do Sul. Logo, os achados sugerem que a escala de Autoeficácia apresenta-se como um instrumento válido e fidedigno para avaliação da autoeficácia em adolescentes.

Estudos de evidências de validade de outras escalas de autoeficácia também indicaram unidimensionalidade da medida, como por exemplo, no estudo das propriedades psicométricas da EAGP em amostra de países como Chile, Espanha, Estados Unidos e Brasil 
(Sbicigo et al., 2012). Scholz, Doña, Sud e Schwarzer (2002) também sustentaram a suposição de que a autoeficácia é um construto unidimensional e universal. A unidimensionalidade de uma medida é considerada desejável num teste, pois o conceito de homogeneidade faz sentido quando se assume avaliar um único atributo. Sua avaliação tem sido referida como central no processo de desenvolvimento de testes psicológicos e deve ser considerada como uma questão de grau, visto que o desempenho humano é multideterminado (Vitória, Almeida, \& Primi, 2006). Portanto, o fato da escala de autoeficácia da BAID-IJ possuir apenas uma dimensão permite entender que o conceito de autoeficácia se mostrou uniforme e coeso, indicando confiabilidade para seu uso em adolescentes.

A pontuação média da amostra na presente pesquisa na escala de autoeficácia foi equivalente ao nível moderado e mostrou que a amostra tende a se perceber capaz de organizar e executar cursos de ação requeridos para produzir determinados resultados (Bandura, 1986), corroborando os achados observados na literatura (Ma et al., 2015; Orcasita et al., 2018; Valois et al., 2015). De modo geral, a autoeficácia significa a crença do indivíduo em sua capacidade de reunir recursos cognitivos, motivacionais, afetivos e comportamentais necessários para alcançar determinado objetivo (Schenell et al., 2015; Sheeran et al., 2016). Portanto, exibir uma pontuação moderada sugere que a amostra, nesse estudo, tem um desempenho adaptativo para lidar com uma determinada situação ou desempenhar uma tarefa.

$\mathrm{Na}$ adolescência, crenças positivas de autoeficácia possibilitam melhor adaptação às mudanças que essa etapa da vida implica. Por exemplo, auxilia na passagem do ambiente familiar para ambientes impessoais, nas descobertas relacionadas à sexualidade e no processo de ajustamento psicológico diante da ocorrência de eventos de vida negativos (Mesurado et al., 2018; Raknes et al., 2017). Assim, a exibição de uma pontuação moderada em autoeficácia por adolescentes sugere que estes administrem de forma ativa e eficaz, diferentes situações e aspectos de sua vida, bem como contribui positivamente para a percepção de sua competência para desempenhar comportamentos específicos. Recomenda-se que processos interventivos, nesse grupo, busquem o desenvolvimento de crenças positivas sobre sua competência pessoal (Tak et al., 2017).

O resultado da correlação de Spearman entre o escore total da Escala de Autoeficácia e o escore total da Escala de Depressão demonstrou associação estatisticamente significativa, moderada e negativa, apontando que quanto menor o nível autoeficácia, maior a presença de sintomas de depressão. Resultados semelhantes também foram observados em outros estudos, (Guerra et al., 2018; Muris et al., 2016; Serra-Taylor \& Irizarry-Robles, 2015). De acordo com Bandura (1986), uma baixa percepção de autoeficácia pode produzir sentimentos de 
apatia, redução da ativação e engajamento comportamental, além de gerar sentimentos de inutilidade e inadequação nos indivíduos, potencializando a ocorrência de depressão.

Neste estudo, se constatou diferença estatisticamente significativa em relação à variável sexo e a autoeficácia, sendo que os meninos apresentaram maiores escores do que as meninas. Outros estudos já evidenciaram resultados semelhantes a este (Muris et al., 2016; Yang et al., 2018). Grande parte das pesquisas que buscou explicar tais diferenças se concentrou em contextos acadêmicos ou escolares, que são tradicionalmente apontados como sendo de domínio masculino ou feminino. Esses estudos evidenciaram, por exemplo, que meninos tendem a relatar crenças de autoeficácia e expectativas maiores do que meninas sobre seu desempenho em matemática e ciências. Enquanto que as meninas apresentaram maior nível de autoeficácia para escrita do que os meninos (Johnson \& Muse, 2017). Essas diferenças podem ser explicadas levando em consideração estereótipos de gênero, expectativas dos pais e validação por seus pares, reforçando crenças de que as meninas são menos competentes que meninos em determinadas tarefas (Bandura et al., 1999).

Acredita-se que as diferenças sociais e de status que promovem mais autonomia, confiança e independência nos meninos, podem explicar as diferenças quanto ao sexo na autoeficácia. Achados empíricos sustentam diferenças por sexo, apontando que os níveis de autoeficácia foram ligeiramente menores nas meninas (Ma et al., 2015; Yang et al., 2018). Além disso, as variáveis relacionadas ao contexto de desempenho de tarefas (domínio específico da autoeficácia) e elementos sociodesenvolvimentais (crenças socialmente construídas) são as hipóteses mais utilizadas para explicar o comportamento diferenciado da autoeficácia por sexo. Acredita-se que os processos de socialização diferenciados entre os sexos, presentes em diferentes culturas, são responsáveis por reforçar crenças de incompetência nas meninas, mesmo em contextos nos quais os desfechos são mais exitosos para elas, como, por exemplo, nas atividades de cuidado (Croft, Schmader, \& Block, 2015). De modo geral, crenças adaptativas de autoeficácia influenciam níveis de saúde em ambos os sexos. Contudo, o achado desta pesquisa sugere a necessidade de intervenções com foco maior no desenvolvimento de crenças positivas de autoeficácia nas meninas.

O resultado da correlação de Spearman demonstrou uma relação estatisticamente significativa e negativa com a variável idade, ainda que fraca, apontando que quanto maior a idade do adolescente, mais baixo foi o nível de autoeficácia. Na literatura, foi identificado apenas um estudo que objetivou investigar a relação entre autoeficácia e a idade do adolescente, sendo constatado que os adolescentes mais velhos apresentaram níveis mais altos 
de autoeficácia comparativamente aos seus colegas mais jovens (Ndika et al., 2009), divergindo, assim, do presente estudo.

A autoeficácia é um construto dinâmico e fluído, que sofre alterações ao longo da vida. Essas alterações dependem de respostas de êxito ou fracasso nas ações empreendidas pelo indivíduo em seu passado. Experiências de sucesso, por exemplo, tendem a aumentar ou fortalecer a percepção sobre eficácia pessoal, ao passo que o fracasso repetido tende a diminuir a crença de autoeficácia (Bandura, 1986). Portanto, o fato de os adolescentes mais velhos terem apresentado menor nível de autoeficácia, comparativamente aos seus colegas mais novos, pode ser entendido como uma percepção possivelmente mais marcada pela vivência de fracassos em ações passadas, o que favoreceria o senso de baixa autoeficácia. Tais fracassos não se constituem, necessariamente, como uma fragilidade, pois fazem parte das experiências de vida de qualquer sujeito e, por esse motivo, podem funcionar como balizas subjetivas pelas quais os indivíduos ponderam a compatibilidade entre sua autoeficácia real e percebida (Schunck, 1995).

Assim, partindo-se do princípio que os adolescentes mais velhos talvez tenham vivenciado mais situações desafiadoras que seus pares mais novos - isso de modo geral e até devido à maior quantidade de anos de vida - essas vivências podem ter funcionado como parâmetros de ajuste entre o quão autoeficaz penso ser e o quanto fui (me percebi) em experiências passadas. Contudo, cumpre ressaltar que a força da correlação aqui detectada foi fraca e a amplitude das idades da amostra foi relativamente pequena (dos 14 aos 19 anos), o que limita a possibilidade de generalização dessa interpretação. Assim, acredita-se que mais estudos são necessários para esclarecer as relações entre autoeficácia e idade, buscando-se, por exemplo, avaliar o papel das experiências de fracasso e sucesso na predição da autoeficácia na adolescência.

Em contrapartida, não foi constatada significância estatística em relação à variável ano escolar. Esse resultado também foi verificado por Joseph et al. (2019). Esse achado revela que, embora as idades médias dos estudantes sejam teoricamente proporcionais ao ano escolar (maior idade, escolaridade mais avançada, por exemplo), o funcionamento da autoeficácia está mais atrelado à idade em si do que à etapa no desenvolvimento escolar, ou seja, a idade revelou um efeito independente do ano escolar nesta pesquisa. Entende-se que as experiências escolares estão diretamente relacionadas ao desenvolvimento de competências no que concernem à aprendizagem, à motivação para a realização, ao funcionamento emocional e relacionamentos sociais, aspectos estes que estão diretamente relacionados ao desenvolvimento de crenças de autoeficácia nessa faixa etária (Roeser \& Eccles, 2000). De 
qualquer modo, como se trata de um dado inicial, sugere-se a averiguação dessas hipóteses interpretativas em futuros trabalhos.

Como limitação desta pesquisa, ressalta-se que a amostra foi do tipo não probabilístico e por conveniência, sem representatividade populacional. Diante disso, é preciso cautela quanto à generalização dos resultados para outros públicos de adolescentes. Contudo, entende-se que estes podem servir como parâmetro de avaliação do construto no público infantojuvenil, visto que se evidenciaram boas qualidades psicométricas, tanto neste quanto no estudo original, feito por Cardoso (2018). Não foi realizada análise de validade concorrente, caminho para se avaliar a adequação do instrumento quanto à validade de critério, importante componente de evidência de validade (Pasquali, 2007). Recomenda-se realizá-la em futuras pesquisas, tais como na relação entre a escala de autoeficácia da BAIDIJ e a EAGP (Schwarzer \& Jerusalem, 1995) ou EAG (Sherer et al., 1982), ambas largamente utilizadas no cenário internacional. Além disso, as Escalas de Autoeficácia e Depressão pertencem à mesma bateria de avaliação. Portanto, os resultados da análise de validade divergente devem ser ponderados. Recomenda-se que futuras pesquisas busquem sanar tal limitação utilizando outros instrumentos. Sugere-se, ainda, a investigação das propriedades psicométricas do instrumento em outras regiões do Brasil, a fim de demonstrar evidências de validade da escala em outros contextos e possibilitar a generalização destes resultados e de atestar a homogeneidade da medida. Por fim, destaca-se que a idade (14 a 19 anos) foi o único parâmetro empregado para definir a amostra deste estudo, não sendo suficiente para caracterizá-la como uma amostra de adolescentes. Torna-se pertinente que futuras pesquisas, nessa faixa etária, busquem critérios mais robustos para delimitação da amostra.

Finalmente, as análises realizadas quanto à AFE demonstraram que a escala de autoeficácia da BAID-IJ apresenta boas evidências de validade com base na sua estrutura interna. A interpretação de seus resultados permite avaliar apropriadamente o construto pretendido, colocando-se para a comunidade científica e profissional como um aparato instrumental útil e de fácil aplicação para futuros estudos, intervenções e avaliações da autoeficácia de adolescentes.

\section{Referências}

Ahn, Y. H., \& Ham, O. K. (2016). Factors associated with medication adherence among medical-aid beneficiaries with hypertension. Western Journal of Nursing Research, 38(10), 1298-1312. doi:10.1177/0193945916651824 
Bandura, A. (1977). Self-efficacy: Toward a unifying theory of behavioral change. Psychological Review, 84(2), 191. doi:10.1037/0033-295X.84.2.191

Bandura, A. (1986). The explanatory and predictive scope of self-efficacy theory. Journal of Social and Clinical Psychology, 4(3), 359-373. doi:10.1521/jscp.1986.4.3.359

Bandura, A., Pastorelli, C., Barbaranelli, C., \& Caprara, G. V. (1999). Self-efficacy pathways to childhood depression. Journal of Personality and Social Psychology, 76(2), 258269. Recuperado de https://www.ncbi.nlm.nih.gov/pubmed/10074708

Bollen, K. A. (1989). A new incremental fit index for general structural equation models. Sociological Methods \& Research, 17(3), 303-316. doi:10.1177/0049124189017003004

Borges, L. S. (2015). Bateria de avaliação de indicadores de depressão infantojuvenil (Tese de Doutorado). Universidade São Francisco, Itatiba, São Paulo, Brasil. Recuperado de http://www.usf.edu.br/ppg/teses.vm?lang=br

Borges, L., Baptista, M. N., \& Serpa, A. L. D. O. (2017). Análise estrutural da bateria de avaliação de indicadores de depresão infantojunenil: Uma abordagem ESEM bifactor. Temas em Psicologia, 25(2), 545-552. doi:10.9788/TP2017.2-08

Capitão, C. G., Scortegagna, S. A., \& Baptista, M. N. (2005). A importância da avaliação psicológica na saúde. Avaliação Psicológica, 4(1), 75-82. Recuperado de http://pepsic.bvsalud.org/scielo.php?script=sci_arttext\&pid=S167704712005000100009

Cardoso, C. (2018). Propriedades psicométricas da bateria de avaliação de indicadores da depressão infantojuvenil (BAID-IJ) (Tese de Doutorado). Universidade São Francisco, Itatiba, São Paulo, Brasil. Recuperado de http://www.usf.edu.br/ppg/teses.vm?lang=br

Croft, A., Schmader, T., \& Block, K. An underexamined inequality: Cultural and psychological barriers to men's engagement with communal roles. Personality and Social Psychology Review, 19(4), 343-370. doi:10.1177/1088868314564789

Damásio, B. F. (2012). Uso da análise fatorial exploratória em psicologia. Avaliação $\begin{array}{llll}\text { Psicológica, } & \text { 11(2), } & \text { 213-228. }\end{array}$ http://pepsic.bvsalud.org/pdf/avp/v11n2/v11n2a07.pdf

Das, J. K., Salam, R. A., Lassi, Z. S., Khan, M. N., Mahmood, W., Patel, V., \& Bhutta, Z. A. (2016). Interventions for adolescent mental health: An overview of systematic reviews. Journal of Adolescent Health, 59(4), 49-60. doi:10.1016/j.jadohealth.2016.06.020 
Ferrando, P. J., \& Lorenzo-Seva, U. (2017). Program FACTOR at 10: Origins, development and future directions. Psicothema, 29(2), 236-240. Recuperado de http://www.psicothema.com/psicothema.asp?id=4389

Gálvez-Nieto, J., Polanco, K., \& Salvo, S. (2017). Propiedades psicométricas de la Escala de Autoconcepto Académico (EAA) en estudiantes chilenos. Revista Iberoamericana de Diagnóstico e Avaliação Psicológica, 43(1), 5-16. doi:10.21865/RIDEP42_69

Gao, S., Zhang, X., \& Xu, X. (2015). A meta-analysis of the relationship between self-esteem and mental health: The sample of Chinese college students. Advances in Psychological Science, 23(9), 1499-1507. doi:10.3724/sp.j.1042.2015.01499

Guerra, C., Farkas, C., \& Moncada, L. (2018). Depression, anxiety and PTSD in sexually abused adolescents: Association with self-efficacy, coping and family support. Child Abuse \& Neglect, 76, 310-320. doi:10.1016/j.chiabu.2017.11.013

Hair, J. F., Black, W. C., Babin, B. J., Anderson, R. E., \& Tatham, R. L. (2009). Análise multivariada de dados. Porto Alegre: Bookman Companhia.

Jager, M. E., Batista, F. A., Perrone, C. M., Santos, S. S., \& Dias, A. C. G. (2014). O adolescente no contexto da saúde pública brasileira: Reflexões sobre o prosad. Psicologia em Estudo, 19(2). doi:10.1590/1413-737221567004

Johnson, I. Y., \& Muse, W. B. (2017). Choice of academic major at a public research university: The role of gender and self-efficacy. Research in Higher Education, 58(4), 365-394. doi:10.1007/s11162-016-9431-1

Jones, P. B. (2013). Adult mental health disorders and their age at onset. The British Journal of Psychiatry, 202(54), 5-10. doi:10.1192/bjp.bp.112.119164

Joseph, C. H., Anikelechi, I. G., \& Marumo, P. (2019). Academic motivation of school going adolescents: Gender and age difference. Gender and Behaviour, 17(1), 12306-12315. Recuperado de https://hdl.handle.net/10520/EJC-15d528d74f

Lorenzo-Seva, U., \& Ferrando, P. J. (2006). Factor: A computer program to fit the exploratory factor analysis model. Behavior Research Methods, 38(1), 88-91. doi:10.1037/a0023353

Ma, Z. W., Zeng, W. N., \& Ye, K. Y. (2015). Gender differences in chinese adolescents' subjective well-being: The mediating role of self-efficacy. Psychological Reports, 116(1), 311-321. doi:10.2466/17.07.PR0.116k15w2

Mao, L., De Wit, J., Adam, P., Post, J. J., Slavin, S., Cogle, A., ... \& Kidd, M. (2018). Beliefs in antiretroviral treatment and self-efficacy in HIV management are associated with 
distinctive HIV treatment trajectories. AIDS and Behavior, 22(3), 887-895. doi:10.1007/s10461-016-1649-6

Mesurado, B., Vidal, E. M., \& Mestre, A. L. (2018). Negative emotions and behaviour: The role of regulatory emotional self-efficacy. Journal of Adolescence, 64, 62-71. doi:10.1016/j.adolescence.2018.01.007

Muris, P. (2001). A brief questionnaire for measuring self-efficacy in youths. Journal of Psychopathology and behavioral Assessment, 23(3), 145-149. doi:10.1023/a:1010961119608

Muris, P., Meesters, C., Pierik, A., \& Kock, B. (2016). Good for the self: Self-compassion and other self-related constructs in relation to symptoms of anxiety and depression in non-clinical youths. Journal of Child and Family Studies, 25(2), 607-617. doi:10.1007/s10826-015-0235-2

Ndika, N. A., Olagbaiye, F., \& Agiobu-Kemmer, I. (2009). Age differences in irrational beliefs, self-efficacy and self-confidence of adolescents in a nigerian secondary school. Psychology and Education, 46(3), 16-26. Recuperado de http://www.psychologyandeducation.net/pae/2012/05/10/age-differences-in-irrationalbeliefs-self-efficacy-and-self-confidence-of-adolescents-in-a-nigerian-secondaryschool/

Nunes, M. F. O. (2008). Funcionamento e desenvolvimento das crenças de auto-eficácia: Uma revisão. Revista Brasileira de orientação profissional, 9(1), 29-42. Recuperado de http://pepsic.bvsalud.org/scielo.php?script=sci_arttext\&pid=S167933902008000100004

Orcasita, L. T., Gil, J. A. M., \& González, T. C. (2018). Autoconcepto, autoeficacia y conductas sexuales de riesgo en adolescentes. Informes Psicológicos, 18(2), 141-168. doi:10.18566/infpsic.v18n2a08

Pasquali, L. (2007). Validade dos testes psicológicos: Será possível reencontrar o caminho. Psicologia: Teoria e Pesquisa, 23(especial). doi:10.1590/S0102-37722007000500019

Raknes, S., Pallesen, S., Bjaastad, J. F., Wergeland, G. J., Hoffart, A., Dyregrov, K., ... \& Haugland, B. S. M. (2017). Negative life events, social support, and self-efficacy in anxious adolescents. Psychological Reports, 120(4), 609-626. doi:10.1177/0033294117699820

Raknes, S., Pallesen, S., Bjaastad, J. F., Wergeland, G. J., Hoffart, A., Dyregrov, K., ... \& Haugland, B. S. M. (2017). Negative life events, social support, and self-efficacy in 
Daiane Nunes, Laís Santos, André Faro, Makilim Nunes Baptista

anxious adolescents. Psychological Reports, 120(4), 609-626. doi:10.1177/0033294117699820

Recber, S., Isiksal, M., \& Koç, Y. (2018). Investigating self-efficacy, anxiety, attitudes and mathematics achievement regarding gender and school type. Anales de Psicología/Annals of Psychology, 34(1), 41-51. doi:10.6018/analesps.34.1.229571

Roeser, R. W., \& Eccles, J. S. (2000). Schooling and mental health. In A. J. Sameroff, M. Lewis, \& S. M. Miller (Orgs.), Handbook of developmental psychopathology (pp. 135-156). Nova York: Kluwer/Plenum.

Sánchez-Teruel, D., \& Robles-Bello, M. A. (2015). Escala de Resiliencia 14 ítems (RS-14): Propiedades Psicométricas de la Versión en Español. Revista Iberoamericana de Diagnóstico y Evaluación-e Avaliação Psicológica, 2(40), 103-113. Recuperado de https://www.aidep.org/sites/default/files/articles/R40/Art10.pdf

Santos, C. M. D. J., \& Faro, A. (2018). Autoeficácia, lócus de controle e adesão ao tratamento em pacientes com diabetes tipo 2. Revista da SBPH, 21, 74-91. Recuperado de http://pepsic.bvsalud.org/scielo.php?script=sci_arttext\&pid=S151608582018000100005

Sawyer, S. M., Afifi, R. A., Bearinger, L. H., Blakemore, S. J., Dick, B., Ezeh, A. C., \& Patton, G. C. (2012). Adolescence: A foundation for future health. The Lancet, 379(9826), 1630-1640. doi:10.1016/S0140-6736(12)60072-5

Sbicigo, J. B., Teixeira, M. A. P., Dias, A. C. G., \& Dell'Aglio, D. D. (2012). Propriedades psicométricas da escala de autoeficácia geral percebida (EAGP). Psico, 43(2), 1. doi:10.15309/17psd180103

Schnell, K., Ringeisen, T., Raufelder, D., \& Rohrmann, S. (2015). The impact of adolescents' self-efficacy and self-regulated goal attainment processes on school performance: Do gender and test anxiety matter?. Learning and Individual Differences, 38, 90-98. doi:10.1016/j.lindif.2014.12.008

Scholz, U., Doña, B. G., Sud, S., \& Schwarzer, R. (2002). Is general self-efficacy a universal construct? Psychometric findings from 25 countries. European Journal of Psychological Assessment, 18(3), 242. doi:10.1027//1015-5759.18.3.242

Schunk, D. H. (1995). Self-efficacy in education and instruction. In J. E Maddux (Ed.), Selfeffi cacy adaptation, and adjustment: Theory, research, and applications (pp. 281303). Greenwich, CT: Information Age Publishing. 
Schwarzer, R., \& Jerusalem, M. (1995). Optimistic self-beliefs as a resource factor in coping with stress. In S. E. Hobfoll, \& M. W. Vries (Eds.), Extreme stress and communities: Impact and intervention (pp. 159-177). Dordrecht, Netherlands: Springer.

Schwarzer, R., \& Luszczynska, A. (2006). Self-efficacy, adolescents' risk-taking behaviors, and health. Self-efficacy beliefs of adolescents, 5, 139-159. Recuperado de http://citeseerx.ist.psu.edu/viewdoc/summary?doi=10.1.1.386.2465

Selzler, A. M., Rodgers, W. M., Berry, T. R., \& Stickland, M. K. (2020). Coping versus mastery modeling intervention to enhance self-efficacy for exercise in patients with COPD. Behavioral Medicine, 46(1), 63-74. doi:10.1080/08964289.2018.1561411

Serra-Taylor, J. A., \& Irizarry-Robles, C. Y. (2015). Protective Factors For Depression In An Elderly Sample Of Puerto Rico: Self-efficacy, Educational Level And Other Sociodemographic Variables. Acta Colombiana de Psicología, 18(1), 125-134. doi:10.14718/ACP.2015.18.1.12

Sheeran, P., Maki, A., Montanaro, E., Avishai-Yitshak, A., Bryan, A., Klein, W. M., ... \& Rothman, A. J. (2016). The impact of changing attitudes, norms, and self-efficacy on health-related intentions and behavior: A meta-analysis. Health Psychology, 35(11), 1178. doi:10.1037/hea0000387

Sherer, M., Maddux, J. E., Mercandante, B., Prentice-Dunn, S., Jacobs, B., \& Rogers, R. W. (1982). The self-efficacy scale: Construction and validation. Psychological reports, 51(2), 663-671. doi:10.2466/pr0.1982.51.2.663

Soysa, C. K., \& Wilcomb, C. J. (2015). Mindfulness, self-compassion, self-efficacy, and gender as predictors of depression, anxiety, stress, and well-being. Mindfulness, 6(2), 217-226. doi:10.1007/s12671-013-0247-1

Tak, Y. R., Brunwasser, S. M., Lichtwarck-Aschoff, A., \& Engels, R. C. (2017). The prospective associations between self-efficacy and depressive symptoms from early to middle adolescence: A cross-lagged model. Journal of Youth and Adolescence, 46(4), 744-756. doi:10.1007/s10964-016-0614-z

Valois, R. F., Zullig, K. J., \& Hunter, A. A. (2015). Association between adolescent suicide ideation, suicide attempts and emotional self-efficacy. Journal of Child and Family Studies, 24(2), 237-248. doi:10826-013-9829-8

Vitória, F., Almeida, L. S., \& Primi, R. (2006). Unidimensionalidade em testes psicológicos: Conceito, estratégias e dificuldades na sua avaliação. Psic: Revista da Vetor Editora, 7(1), 1-7. Recuperado de 
http://pepsic.bvsalud.org/scielo.php?script=sci_arttext\&pid=S167673142006000100002

World Health Organization [WHO]. (2014). Health for the world's adolescents: A second chance in the second decade. Geneva: WHO.

Yang, X., Lau, J. T., \& Lau, M. C. (2018). Predictors of remission from probable depression among Hong Kong adolescents-A large-scale longitudinal study. Journal of Affective Disorders, 229, 491-497. doi:10.1016/j.jad.2017.12.080

\section{Endereço para correspondência}

\section{Daiane Nunes}

Av. Marechal Rondon, 210 quadra 4 bl. 2 apto 201, Jd. Rosa Elze, São Cristóvão - SE, Brasil. CEP 49100-000

Endereço eletrônico: daianenunesufs@gmail.com

\section{Laís Santos}

Rua da Abolição, 949 apto 62B, Ponte Preta, Campinas - SP, Brasil. CEP 13041-445

Endereço eletrônico: laiss.santos9597@gmail.com

\section{André Faro}

Avenida Marechal Rondon, s / n, Jd. Rosa Elze, São Cristóvão - SE, Brasil. CEP 49100-000

Endereço eletrônico: samazo@hotmail.com

\section{Makilim Nunes Baptista}

Rua Waldemar César da Silveira, 105, Jardim Cura D'ars, Campinas - SP, Brasil. CEP 13045-510

Endereço eletrônico: makilim01@gmail.com

Recebido em: 05/04/2019

Reformulado em: 14/05/2020

Aceito em: 01/06/2020

\section{Notas}

* Mestre em Psicologia pelo Programa de Pós-Graduação em Psicologia (PPGPSI) da Universidade Federal de Sergipe (UFS).

** Doutoranda em Psicologia pela Pontifícia Universidade Católica de Campinas (PUC-Campinas). Bolsista CAPES.

*** Doutor em Psicologia. Docente do PPGPSI da UFS. Pesquisador Produtividade pelo CNPq (Nível 2).

**** Doutor em Psicologia. Docente do Programa de Pós-Graduação Stricto-Sensu em Psicologia da Universidade São Francisco (USF). Pesquisador Produtividade pelo CNPq (Nível 1).

Financiamento: apoio financeiro da Fundação de Apoio à Pesquisa e à Inovação Tecnológica do Estado de Sergipe (FAPITEC/SE). 
Daiane Nunes, Laís Santos, André Faro, Makilim Nunes Baptista

Este artigo de revista Estudos e Pesquisas em Psicologia é licenciado sob uma Licença Creative Commons Atribuição-Não Comercial 3.0 Não Adaptada. 